1

2

3

4

5

6

7

\title{
Proteomic characterization of human coronary thrombus in patients with ST-segment Elevation Acute Myocardial Infarction
}

\author{
Sergio Alonso-Orgaz ${ }^{1}$, Rafael Moreno-Luna ${ }^{1}$, Juan A. López ${ }^{2}$, Felix Gil-Dones ${ }^{1}$, Luis \\ R. Padial ${ }^{3}$, Jose Moreu ${ }^{4}$, Fernando de la Cuesta ${ }^{1}$, Maria G. Barderas ${ }^{1}$.
}

1, Department of Vascular Physiopathology, Hospital Nacional de Paraplejicos SESCAM, Toledo. 2, Unidad de Proteomica, CNIC, Madrid. 3, Cardiology Department, Hospital Virgen de la Salud, SESCAM, Toledo. 4, Hemodynamics Department, Hospital Virgen de la Salud, SESCAM, Toledo.

*, Both senior authors contributed equally to this work.

Corresponding author: Dr. Fernando de la Cuesta, PhD. Laboratorio de Fisiopatologia Vascular, Edificio de Terapia, $2^{a}$ planta. Hospital Nacional de Paraplejicos, SESCAM, 45071 Toledo, Spain. e-mail: ferdela@ sescam.jccm.es FAX number: (+34) 925247745 
ABSTRACT

Acute Myocardial Infarction with ST-segment elevation (STEMI) initiates with intraluminal thrombosis and results in total occlusion of the coronary artery. To date, characterization of the coronary thrombus proteome in STEMI patients has not been yet accomplished. Therefore, we aimed to perform an in-depth proteomic characterization of the coronary thrombus by means of three different approaches: 2-DE followed by mass spectrometry (MALDI MS/MS), 1-DE combined either with liquid chromatography coupled to mass spectrometry in a MALDI TOF/TOF (LC-MALDIMS/MS), or in a LTQ-Orbitrap (LC-ESI-MS/MS). This approach allowed us to identify a total of 708 proteins in the thrombus. Expression in human coronary thrombi $(n=20)$ of 14 proteins was verified, and the expression of fibrin and 6 cell markers (platelets, monocytes, neutrophils, eosinohils, T-cells and B-cells) quantified by SRM. A positive correlation of 5 proteins (fermitin homolog 3, thrombospondin-1, myosin-9, beta parvin and ras-related protein Rap-1b) with CD41 was found, pointing out the activation of a focal adhesion pathway within thrombi platelets. DIDO1 protein was found to correlate negatively with thrombus fibrin, and was found up-regulated in the plasma of these STEMI patients, which constitutes an important starting point for further analyses in the search for biomarkers of thrombosis.

41 Keywords: human coronary artery, acute myocardial infarction, focal adhesion, thrombosis, DIDO1. 
ST-segment elevation myocardial infarction (STEMI) is caused by the rupture or the erosion of a vulnerable atherosclerotic plaque, initiating with intraluminal thrombosis and resulting in total occlusion of the coronary artery [1]. Several circulating cell types such as platelets, erythrocytes and monocytes, among others, as well as plasma molecules, modulate the final consequences of plaque disruption, contributing differently to the atherosclerotic process and therefore to subsequent thrombus formation [2, 3]. These circulating cells have been widely studied under different pathophysiological states [4,5] focusing on their cellular protein expression and their modifications during the development of cardiovascular episodes [3, 6]. In contrast, thrombus studies have been limited, mainly due to the difficult accessibility to the thrombotic material. Currently, the extraction of occlusive thrombi is achieved by an aspiration catheter, which is introduced in the coronary arterial tree. The use of this methodology has allowed obtaining coronary thrombi with acute phase characteristics from patients suffering from myocardial infarction [7]. The majority of studies performed in aspirated material are histological, based on the presence of different cell types in the coronary thrombus $[8,9]$ or the expression of different thrombosis-related proteins detected by immunohistochemistry (IHC) [10]. In a recent work, Silvain et al. studied composition of the coronary thrombus in STEMI patients by means of scanning electron microscopy. Results obtained highlighted a dynamic evolution of thrombi composition with ischemia time, with a significant decrease in platelet number and a significant increase in fibrin [11].

The integrin alpha-IIb/beta-3 (CD41) is a receptor present in platelets, which binds fibronectin, plasminogen, prothrombin, thrombospondin and vitronectin, as well as fibrinogen and its cleavage product fibrin. CD41 is responsible for platelet 
aggregation and interaction with extracellular matrix (ECM) and other cells upon activation [12]. Although the role of platelets in thrombosis has been deeply characterized at the molecular level by means of numerous in vitro studies [13], the molecular mechanisms underlying platelet activation and focal adhesion within coronary thrombi have not been to date clearly elucidated.

Concerning clinical proteomics, which main objective is identifying proteins involved in a disease in a defined biological system, the analysis of the protein content of the human coronary thrombus in the context of STEMI is still a pending issue. The proteomic methodology has been already applied to describe the proteome of platelets[14], erythrocytes [15] and monocytes [16] as well as to define protein profiles of such cells associated to acute coronary syndrome [3,6] and to characterize the proteome of the atherosclerotic plaque tissue $[17,18]$. The analysis of the thrombus proteome may reveal the contribution of the aforementioned described factors, involved in the atherothrombotic process leading to thrombus formation. Previous thrombi proteomic studies have been performed with cells isolated from venous thrombi [19] or have only focused on substances released by aortic artery thrombi [20]. Therefore, to date, no comprehensive proteomic characterization of the coronary thrombus has been conducted. The objective of the present study was to identify, through a global proteomics approach, the proteome of the coronary thrombus from patients with STEMI, as well as to build a 2-DE map of this sample, as a reference for future comparative studies. Moreover, 14 proteins were analyzed by SRM together with 6 cell markers (CD41, CD3, CD14, CD19, ELNE and PERE) and fibrin, and correlation analyses between all molecules allowed describing a novel methodology to link protein expression with cellular and ECM measures, therefore contextualizing protein changes with the addition of thrombus composition information. 
The coronary thrombus extracted just after a STEMI reflects the dynamic process triggered within the artery leading to myocardial infarction, thereby containing proteins locally expressed by the activated cells present, which may be released to the blood and that could have potential use as biomarkers. Hence, the protein DIDO1 was found upregulated in the plasma of these STEMI patients, which constitutes an important starting point for further analyses in the search for biomarkers of thrombosis.

\section{MATERIALS AND METHODS}

\subsection{Patient population and thrombus collection}

Twenty patients with STEMI were recruited at the Hemodynamic Service of Hospital Virgen de la Salud of Toledo within 12 hours of chest pain onset. By protocol all patients were pretreated with AAS and clopidogrel. Unfractioned heparin and Abxicimab@ adjusted to body weight were used during examination in the catheterization laboratory. The occluded segment was crossed with hydrophilic wire and the patients were subjected to percutaneous intracoronary thrombectomy during primary angioplasty. Thrombectomy was performed with the $6 \mathrm{~F}$ catheter Export ${ }^{\circledR}$ (Medtronic Iberica). Aspirated blood and intracoronary thrombus material were collected in a collection bottle provided with a filter. Shortly after extraction, the thrombi tissue was cleaned with saline solution $(0.9 \%)$ to reduce plasma contaminants. The aspirated thrombi material was equally divided in two fragments, one of which was embedded in OCT (Sakura Finetek USA Inc.; Torrance, CA) and the other one frozen and stored at $-80^{\circ} \mathrm{C}$ until used for proteomic analysis. This study was carried out in accordance with the recommendations of the Helsinki Declaration and it was approved by the ethics committee at the Hospital "Virgen de la Salud" (Toledo, Spain). Signed informed consent was obtained from all subjects prior to their inclusion in the study. Clinical characteristics of selected patients are 
shown in Table 1. The first four thrombi were used for a comprehensive proteomic analysis of human coronary proteome and all twenty thrombi were employed for further analyses.

EDTA coagulated plasma from 17 out of the 20 patients, and 16 healthy subjects with matched clinical characteristics (no significant differences were observed for age, gender and cardiovascular risk factors, Supplementary Table 1), was collected and stored at $-80^{\circ} \mathrm{C}$ until used.

\subsection{Histopathological analysis}

The four thrombi used for the characterization of human coronary thrombus proteome were subjected to histopathological analysis, in order to validate collection procedure. Aspirated material was embedded in OCT, serially cut in $5 \mu \mathrm{m}$ and mounted on glass slides. The sections were stained with Hematoxilin and Eosin (H\&E) for light microscopy. Immunohistochemical analysis was performed with antibodies against CD41, fibrinogen and neutrophil elastase (all from Abcam). After blocking non-specific reactions with $10 \%$ goat normal serum (Abcam), avidin $0.001 \%$ and biotin $0.001 \%$ in PBS-T $0.05 \%$, sections were incubated with primary antibodies at room temperature (RT) for 1 hour. Secondary antibody conjugated with biotin, streptavidin-peroxidase and DAB used for all immunostainings was from Mouse and Rabbit Specific HRP/DAB (ABC) Detection IHC kit (Abcam).

\subsection{Protein extraction from thrombi}

Frozen aspirated thrombi were ground into a powder in liquid nitrogen with a mortar. An amount of 0.1-0.3 g of this powder was resuspended in $200 \mu \mathrm{L}$ of protein extraction buffer (7M Urea, 2M Thiourea, 4\% CHAPS, PMSF 1mM). The homogenate was sonicated $5 \mathrm{~min}$, centrifugated at 21,000g (5840R Eppendorf) for $15 \mathrm{~min}$ at $4^{\circ} \mathrm{C}$, and 
the supernatant was separated from the pellet of tissue debris. The protein concentration was determined by a Bradford-Lowry method (Bio-Rad protein assay).

\subsection{Unidimensional and two-dimensional gel electrophoresis (1-DE and 2-DE)}

An amount of $36 \mu \mathrm{g}$ of a pool of protein extracts from 4 patients was loaded in 10\% SDS-polyacrylamide gels for unidimensional electrophoresis (1-DE). The lane in the 1-DE gel was divided into 10 gel slices that were manually excised for LC-MALDIMS/MS (Figure 1). For LC-ESI-MS/MS analysis, the electrophoresis was stopped when the sample had barely passed the resolution gel, so that it was concentrated in a unique band [21]. The band in the 1-DE gel was manually excised and afterwards digested.

All chemicals and instruments used in 2-DE gels have been previously described [22]. Thrombi extracts were delipidated by adding ice-cold tri- $n$-butylphosphate: acetone: methanol $(1: 12: 1)$ to a final acetone concentration of $80 \%$ and incubated at $4{ }^{\circ} \mathrm{C}$ for 90 min according to Leppeda et al. [18]. Precipitates were re-solubilized in the same extraction buffer by repeated sonications. An amount of $75 \mu \mathrm{g}$ from each protein extract from the 4 patients was mixed and dialysed against $20 \mathrm{mM}$ Tris buffer using Mini dialysis Kit 1kDa cut-off (GE Healthcare). Subsequently, $300 \mu \mathrm{g}$ of the pooled protein extract was cleaned with 2-D Clean-up Kit (GE-Healthcare) and resuspended in rehydration buffer (7M Urea, 2M Thiourea, 4\% CHAPS, 1\% Ampholites and 1\% TBP). Isoelectric focusing (IEF) was performed in a PROTEAN IEF Cell unit (Bio-Rad). The IPG strip (17cm and $\mathrm{pH} 4-7$, Bio-Rad) was actively rehydrated at $20^{\circ} \mathrm{C}$ for $12 \mathrm{~h}$ at $50 \mathrm{~V}$ to enhance protein uptake and then the voltage was increased according to the following program: $500 \mathrm{~V}$ for $1 \mathrm{~h}, 1000 \mathrm{~V}$ for $1 \mathrm{~h}, 1000-8000 \mathrm{~V}$ in $1 \mathrm{~h}$ (gradient), $8000 \mathrm{~V}$ for a total $50.000 \mathrm{~V} / \mathrm{h}$. Following IEF, IPG strips were equilibrated in $6 \mathrm{M}$ urea, $50 \mathrm{mM}$ Tris- $\mathrm{HCl}$ $\mathrm{pH} 8.8,30 \%$ glycerol, $4 \%$ SDS, first with DTT $(1 \% \mathrm{~m} / \mathrm{v})$ and subsequently with IAA $(2.5 \% \mathrm{~m} / \mathrm{v})$, for $20 \mathrm{~min}$. Second dimension (SDS-PAGE in $12 \%$ polycarylamide gels) 
was performed in an Ettan Dalt Six system (GE Healthcare) overnight at 1W/gel and $25^{\circ} \mathrm{C}$ overnight. Gels were fixed and stained using Silver Staining kit (GE Healthcare) according to the manufacturer's indications and they were then scanned with GS-800 Calibrated Densitometer (Bio-Rad).

\subsection{Protein digestion}

The digestion of gel slices and spots was performed according to Schevchenko et al. [23] with minor modifications using the Ettan Digester (GE Healthcare): gel slices from 1-DE gel and spots excised from 2-DE gel were incubated with $10 \mathrm{mM}$ DTT (Sigma Aldrich) in $50 \mathrm{mM}$ ammonium bicarbonate (99\% purity; Scharlau) for 30min at $56^{\circ} \mathrm{C}$ and after reduction, alkylation with $55 \mathrm{mM}$ iodoacetamide (Sigma Aldrich) in 50 $\mathrm{mM}$ ammonium bicarbonate was carried out for 20min at RT. Gel plugs were washed with $50 \mathrm{mM}$ ammonium bicarbonate in 50\% methanol (gradient, HPLC grade, Scharlau), rinsed in acetonitrile (gradient, HPLC grade, Scharlau) and dried in a Speedvac. Dry gel pieces were covered with sequencing grade modified porcine trypsin (Promega, Madison, WI, USA) at a final concentration of $20 \mathrm{ng} / \mu \mathrm{L}$ in $20 \mathrm{mM}$ ammonium bicarbonate. After digestion at $37^{\circ} \mathrm{C}$ overnight, peptides were extracted with $60 \%$ acetonitrile $(\mathrm{ACN})$ in $0.5 \%$ trifluoroacetic acid (99.5\% purity; Sigma Aldrich) and dried in a Speedvac. The digested samples from 1-DE gel were resuspended in $20 \mu \mathrm{L}$ [98\% water with $2 \%$ trifluoroacetic acid and $2 \% \mathrm{ACN}]$ for LC separation.

\subsection{MALDI-MS/MS analysis of 2-DE spots}

A volume of $0.5 \mu \mathrm{L}$ from each 2-DE spot digestion solution was deposited using the thin layer method, onto a 384 Opti-TOF 123x81 mm MALDI plate (Applied Biosystems) and allowed to dry at room temperature. The same volume of matrix, $3 \mathrm{mg} / \mathrm{mL} \alpha$-cyano-4-hydroxycinnamic acid (CHCA, Sigma Aldrich) in 60\% acetonitrile, $0.5 \%$ trifluoroacetic acid, was applied on every sample in the MALDI plate. MALDI- 
MS(/MS) data were obtained in an automated analysis loop using a 4800 Plus MALDI

TOF/TOF Analyzer (Applied Biosystems). Automated analysis of mass data was performed using the 4000 Series Explorer Software version 3.5.3 (Applied Biosystems). MALDI-MS and MS/MS data were combined through the GPS Explorer Software Version 3.6 to search a nonredundant protein database (Swissprot 2011_11) using the Mascot software version 2.2 (Matrix Science), with $50 \mathrm{ppm}$ precursor tolerance, 0.6 Da MS/MS fragment tolerance and carbamidomethyl cysteine as fixed modification, oxidized methionine as variable modification and allowing 1 missed cleavage. MALDIMS(/MS) spectra and database search results were manually inspected in detail using higher. collector (Dionex). CHCA (Sigma-Aldrich) was used as MALDI matrix. The contact start signal was send to the Probot after $10 \mathrm{~min}$ LC run time to start the fractionation. 
218 Biosystems) using a 32 x 52 geometry (1664 spots per plate). MS and MS/MS analysis

219 of offline spotted peptide samples were performed using the Applied Biosystems 4800

220 plus MALDI TOF/TOF Analyzer mass spectrometer. Peptide and protein identifications

221 were performed using ProteinPilot ${ }^{\mathrm{TM}}$ Software V 2.0.1 (Applied Biosystems) and the

222 Paragon algorithm. Each MS/MS spectrum was searched against the SwissProt 2011_11

223 database, with the fixed modification of carbamidomethyl cysteine parameter enabled.

224 Other parameters such as the tryptic cleavage specificity, the precursor ion mass accuracy and the fragment ion mass accuracy, are MALDI 4800 built in functions of the ProteinPilot software. Although this software automatically accepts all peptides with an identification confidence level $>1 \%$, only proteins having at least one peptide above the $90 \%$ confidence level were initially recorded. Search against a concatenated database containing both forward and reversed sequences (decoy search) enabled the false discovery rate to be kept below $1 \%$.

\subsection{LC-ESI-MS/MS data analysis}

Peptides were injected onto a C-18 reversed phase (RP) nano-column (100 $\mu \mathrm{m}$

I.D. and $12 \mathrm{~cm}$, Mediterranea sea, Teknokroma) and analyzed in a continuous acetonitrile gradient consisting of $0-43 \% \mathrm{~B}$ in $140 \mathrm{~min}, 50-90 \% \mathrm{~B}$ in $1 \mathrm{~min}(\mathrm{~B}=95 \%$ acetonitrile, $0.5 \%$ acetic acid). A flow rate of $300 \mathrm{nl} / \mathrm{min}$ was used to elute peptides from the RP nano-column to a PicoTip ${ }^{\mathrm{TM}}$ emitter nano-spray needle (New Objective, Woburn, MA) for real time ionization and peptide fragmentation on an LTQ-Orbitrap XL ETD mass spectrometer (Thermo Fisher, San José, CA, USA). An enhanced FTresolution spectrum (resolution=60000) followed by the MS/MS spectra from most intense five parent ions were analyzed along the chromatographic run (180 $\mathrm{min})$. Dynamic exclusion was set at $0.5 \mathrm{~min}$. 

Discoverer version 1.0 (Thermo Fisher Scientific). All MS/MS samples were analyzed using Sequest (Thermo Fisher Scientific; version 1.0.43.2) and X! Tandem (The GPM, thegpm.org; version 2007.01.01.1). X! Tandem was set up to search a subset of the human database assuming the digestion enzyme trypsin. Sequest was set up to search human_ref.fasta, (39414 entries) assuming trypsin digestion. Sequest and X! Tandem were searched with a fragment ion mass tolerance of $0.80 \mathrm{Da}$ and a parent ion tolerance of $10.0 \mathrm{ppm}$. Oxidation of methionine, acetylation of lysine and phosphorylation of serine, threonine and tyrosine were specified in Sequest and X! Tandem as variable modifications. Scaffold (version Scaffold_3_00_03, Proteome Software Inc., Portland, OR) was used to validate MS/MS based peptide and protein identifications. Protein probabilities were assigned by the Protein Prophet algorithm. Protein identification was performed establishing protein and peptide probability greater than $99 \%$ and $95 \%$, respectively. Proteins that contained similar peptides and could not be differentiated based on MS/MS analysis alone were grouped to satisfy the principles of parsimony.

\subsection{Bioinformatics analysis of identified proteins}

For a functional examination of the identified proteins, the list of 708 proteins identified was implemented on the on-line software David Bioinformatics Resources 6.7 (NIH) [24] and Functional Annotation Tool was used to search for enriched Gene Ontology (biological process and molecular function) categories, as well as for pathway analysis, using KEGG Pathways database. Functional annotation clustering was performed in order to avoid redundancy of enriched categories and pathways (data not shown). Functional annotation chart for each search and the proteins included in every group are provided in the supplementary material. 
Protein samples were reduced with $100 \mathrm{mM}$ DTT in $50 \mathrm{mM}$ ammonium

bicarbonate (Scharlau) for $30 \mathrm{~min}$ at $37^{\circ} \mathrm{C}$, and alkylated for $20 \mathrm{~min}$ at room temperature (RT) with $550 \mathrm{mM}$ iodoacetamide in $50 \mathrm{mM}$ ammonium bicarbonate. The proteins were then digested in $50 \mathrm{mM}$ ammonium bicarbonate, $15 \%$ acetonitrile (Scharlau) with sequencing grade modified porcine trypsin at a final concentration of 1:50 (trypsin:protein). After overnight digestion at $37{ }^{\circ} \mathrm{C}, 2 \%$ formic acid (Sigma Aldrich) was added and the samples were cleaned with Pep-Clean spin columns (Pierce) according to the manufacturer's instructions. Tryptic digests were dried in speed-vac and resuspended in $2 \%$ acetonitrile, $2 \%$ formic acid (FA) prior to MS analysis.

The LC-MS/MS system consisted of a TEMPO nano LC system (Applied Biosystems) combined with a nano LC Autosampler coupled to a modified triple quadrupole (4000 QTRAP LC/MS/MS, Applied Biosystems). Three replicate injections ( $2 \mu \mathrm{g}$ of protein in $4 \mu \mathrm{L})$ were performed per sample in the mobile phase A $(2 \%$ ACN/98\% water, $0.1 \%$ FA) at a flow rate of $10 \mu \mathrm{L} / \mathrm{min}$ for $5 \mathrm{~min}$. Peptides were loaded onto a $\mu$-Precolumn Cartridge (Acclaim Pep Map 100 C18, $5 \mu \mathrm{m}, 100 \AA$; $300 \mu \mathrm{m}$ i.d. X 5mm, LC Packings) to preconcentrate and desalt samples. Reversed-phase liquid chromatography (RPLC) was performed on a C18 column (Onyx Monolithic C18, 150 x 0.1mm I.D., Phenomenex) in a gradient of phase A and phase B (98\% ACN $/ 2 \%$ water, $0.1 \%$ FA). The peptides were eluted at a flow rate of $300 \mathrm{nl} / \mathrm{min}$ in a continuous acetonitrile gradient: $2 \%-15 \%$ B for $2 \mathrm{~min}, 15 \%-50 \%$ B for $38 \mathrm{~min}, 50$ to $90 \%$ B for 2 min and 90\% B for 3 min. Both the TEMPO nano LC and 4000 QTRAP system were controlled by Analyst Software v.1.4.2.

Theoretical SRM transitions were designed using MRMpilot software v1.1 (ABSciex), with the following settings: Enzyme $=$ trypsin, missed cleavages $=0$; 
modifications in peptide $\leq 3$; charge states $=+1$ from 300 to $600 \mathrm{Da},+2$ from 500 to $2000 \mathrm{Da},+3$ from 900 to $3000 \mathrm{Da},+4$ from 1600 to $4000 \mathrm{Da},+5$ from 2400 to 10000

Da; studied modification $=$ none; fixed modifications $=$ carboxyamidomethylation; variable modifications $=$ none; $\min$. number of amino acids $\geq 5$; $\max$. number of amino acids $\leq 30$; ignore multiple modification sites; 3 transitions per peptide (Supplementary Table 2). A pool containing a mixture of all the samples was digested as described previously and analyzed in the 4000QTrap using a MIDAS acquisition method that included the theoretical transitions. Transitions were selected when the three co-eluting peaks (corresponding to the three transitions of the same peptide) had a signal-to-noise ratio over 5 and the MS/MS data matched the theoretical spectrum for that peptide. Collision energy was optimized to obtain the maximum transmission efficiency and sensitivity for each SRM transition. Transitions monitored during individual sample analyses were acquired at unit resolution in both Q1 and Q3, with dwell times of $50 \mathrm{~ms}$ resulting in cycle times of $1.8 \mathrm{~s}$. The IntelliQuan algorithm included in Analyst 1.4.2

We used the MARS-14 column (Agilent Technologies) to remove the 14 most transthyretin, C3 and 92-99\% fibrinogens). Human plasma $(20 \mu \mathrm{L})$ from STEMI patients $(\mathrm{n}=17)$ and controls $(\mathrm{n}=16)$ was five-fold diluted in "Buffer A" (Agilent Technologies) and spun in a microfuge for 1 min through a $0.22 \mu \mathrm{m}$ spin filter tube at maximum speed (about 16,000 g). The sample was then injected into a 1200 series 
HPLC System (Agilent Technologies) and chromatography was performed according to manufacturer's instructions. After chromatography, the aliquots of the flow-through fractions containing low-abundance proteins were combined and desalted using centrifugal filter devices with a $3 \mathrm{kDa}$ cut off (Amicon Ultra, Millipore). These samples were stored at $-80{ }^{\circ} \mathrm{C}$ prior to analysis

bands were measured using a GS-800 Calibrated Densitometer (Bio-Rad) A

Kolmogorov-Smirnov test demonstrated the normal distribution of the population 
was used for band intensity analysis.

Correlations between proteins were evaluated by calculating Pearson productmoment correlation coefficient of every transition from every peptide analyzed for each protein with respect to every transition of the peptides of the other proteins. If the results of 1 out of the 3 assayed transitions were divergent from the other two, this transition was not considered. While evaluating correlations, when 2 or more peptides were measured, the most significant peptide was selected, while the second one was used as a qualifier for the correlation, which was rejected if this second peptide had divergent results. Pearson's correlation coefficient (r) was calculated as a mean of all coefficients from all transitions from the most significant peptide-to-peptide correlation. Correlation $\mathrm{p}$ value was expressed as $<0,05 ;<0,01 ;<0,001$ when all $\mathrm{p}$ values from all transitions from the most significant peptide-to-peptide correlation were lower than the specified values. When any of the transition-to-transition correlations was not significant, but close to signification, the greater transition-to-transition $p$ value of the most significant peptide-to-peptide correlation was considered. For linear regression representation, the most significant transition was selected in each case.

\section{RESULTS}

Three complementary proteomic approaches based on in-gel separation of proteins (2DE-MALDI-MS/MS) and on liquid-chromography separation of peptides (LC-MALDI-MS/MS and LC-ESI-MS/MS) were combined to perform a comprehensive characterization of the human coronary thrombus proteome, by analyzing a pool of 4 different thrombi from STEMI patients.

\subsection{Histology of thrombotic material}

$\mathrm{H} \& \mathrm{E}$ and immunostaining of thrombotic material allowed approving the collection procedure, since obtained tissue was composed of platelets (Figure 2C), 
erythrocytes (Figure 2B), fibrin (Figure 2D, see positive fibrin fibers and fibrinogenexpressing platelets) and neutrophils (Figure 2E).

\subsection{2-DE-MALDI-TOF/TOF}

Three technical replicates of human coronary thrombi gels were run showing minimal variability between runs, which confirmed reproducibility of the method. After PD-Quest software analysis (BioRad), more than 1000 spots were detected in coronary thrombi gels (Figure 3). An amount of 235 spots were excised from a 2-DE silverstained gel, digested and the resultant tryptic peptides were deposited in a MALDI plaque and analyzed in a 4800 Plus MALDI-TOF/TOF Analyzer (Applied Biosystem). All but 11 spots (224) were identified, corresponding to 81 unique proteins, 38 of which were represented by more than 1 spot (Figure 3). Supplementary Table 3 summarizes these identified proteins, their molecular weight, isoelectric point, subcellular location and function.

\subsection{Liquid-Chromatography Mass Spectrometry (LC-MS/MS)}

To improve the number of proteins identified by 2-DE MALDI-TOF/TOF, a LCMS/MS analysis was carried out using two different methodologies: 1-DE LC-MALDIMS/MS and LC-ESI-MS/MS.

Using LC-MALDI-MS/MS approach, a total of 4,991 peptides were identified, which corresponded to 13,254 spectra. After data grouping and filtration, 372 proteins were identified (cut off $>1.3$ and $>95 \%$ confidence interval, C.I.) and their theoretical MW and pI, subcellular location and function are shown in Supplementary Table 4.

LC-ESI-MS/MS analysis allowed identifying a total of 2,520 unique peptides, which corresponded to 7,580 spectra. After data grouping and filtration, 467 proteins 
were identified (>95\% C.I.) and their theoretical MW, subcellular location and function are shown in Supplementary Table 5.

\subsection{Characterization and classification of identified proteins}

A total of 708 unique proteins were identified with the three methodologies employed (Supplementary Table 6), 81 proteins corresponding to 235 spots were identified by 2-DE MALDI-TOF/TOF; 372 proteins by 1-DE LC-MALDI-MS/MS and 467 proteins by LC-ESI-MS/MS. Venn diagram showing the number of proteins identified by these methods can be observed in Figure 4A. The 138 proteins identified by both LC-MS/MS approaches and the 46 identified by all methodologies are compiled in Suplementary Table 4.

Proteins were categorized in seven functional groups (Figure 4B); "Cell differentiation", "Metabolism", "Redox State and Apoptosis", "Regulation and transport", "Immune response and acute phase", "Structural and cell adhesion" and "Other", based on NCBI and UniProt data base information. Furthermore, the proteins were also classified by their subcellular location (Figure 4C).

Functional analysis performed with David 6.7 software for Gene Ontology (GO) categories "molecular function" and "biological process" reported a significant enrichment of cytoskeleton proteins. Other enriched GO categories were: "Blood coagulation", "Response to wounding", as well as "Nucleotide binding", which showed a substantial contribution of "ATP binding" proteins (105 out of the 163 proteins) (Supplementary Figure 1). On the other hand, remarkable pathways found enriched in the protein list were "Regulation of actin cytoskeleton", "Focal adhesion" (Figure 5), “Tight junction”, “Gap junction”, “Adherens junction”, “Leukocyte transendothelial migration", "Glycolysis/gluconeogenesis" and "Coagulation cascade" (Supplementary Figure 2). 

thrombi

A subset of 14 proteins identified by at least 2 out of the 3 proteomic approaches, with functional relevance for thrombus formation, were selected for further analyses: fermitin family homolog 3 (FERM3), death-inducer obliterator 1 (DIDO1), flavin reductase (NADPH), carbonic anhydrases 1, 2 and 3, calreticulin, catalase, multimerin-1 (MMRN1), myosin-9 (MYH9), beta-parvin (PARVB), ras-related protein Rap-1b (RAP1B), titin and thrombospondin-1 (TSP1). This proteins were analyzed by SRM in all the collected thrombi $(n=20)$ together with 6 cell markers: CD41 (platelets), CD3 (T lymphocytes), CD14 (monocytes), CD19 (B lymphocytes), neutrophil elastase and eosinophil peroxidase; and fibrin (analyzed peptides and transitions are displayed in Supplementary Table 2). Optimization experiments performed with a pool of all 20 samples allowed detecting all proteins, verifying its expression within the human coronary thrombus of STEMI patients (chromatographic peaks of every transition are provided in Supplementary Figure 3). Every protein was quantified using 3 transitions of 2 peptides, except for fibrin (3 peptides: 2 of chain A, 1 of chain B), CD3 (3 peptides: 2 of chain gamma and 1 of chain delta), and CD14 and FERM3, in which only 1 peptide could be measured. Moreover, correlation analyses between all studied proteins were performed, in order to link protein expression of the analyzed proteins with the cell and ECM (fibrin) markers. Significant and close to significance correlations are displayed on Supplementary Table 7.

Concerning cell markers evaluated, T-cell marker CD3 correlated positively with monocyte and B-cell markers (CD14: $\mathrm{r}=0.98$; $\mathrm{p}$ value $<0.001$ and CD19: $\mathrm{r}=0.57$; $\mathrm{p}$ value $<0.05)$. A negative correlation of ELNE with CD3 ( $r=-0.49$; p value $<0.05)$ and CD14 ( $\mathrm{r}=-0.49$; $\mathrm{p}$ value $<0.05$ ) was also found. A positive correlation was found for ELNE with PERE (r=0.68; $\mathrm{p}$ value $<0.01$ ). A positive correlation of CD14 with CD19 
was close to signification $(\mathrm{r}=0.42 ; \mathrm{p}$ value $<0.097)$.

A group of 5 proteins were found to correlate positively with the platelet marker CD41: FERM3 (r=0.94; p value<0.001), MYH9 $(\mathrm{r}=0.89 ; \mathrm{p}$ value<0.001), TSP1 $(\mathrm{r}=0.96$; $\mathrm{p}$ value $<0.001)$ PARVB $(\mathrm{r}=0.96 ; \mathrm{p}$ value $<0.01)$ and $\mathrm{RAP1B}(\mathrm{r}=0.95 ; \mathrm{p}$ value $<0.001)$ (Figure 6A). Furthermore, all these proteins showed a positive correlation with the other 4 proteins, with a Pearson's coefficient above 0.76 and a palue $<0,001$ (Supplementary Table 7). Multimerin-1 (MMRN1) was found to correlate positively with neutrophil and eosinophil markers (ELNE: r=0.98; p value<0,001); PERE: $r=0.66$; $\mathrm{p}$ value $<0.01)$ and negatively with $\mathrm{CD} 3(\mathrm{r}=-0.52$; $\mathrm{p}$ value $<0.05)$. A negative correlation with monocytes very close to signification was also found $(r=-0.42 ; p$ value $<0.08)$.

Fibrin showed a negative correlation with DIDO1 ( $r=-0.50, \mathrm{p}$ value $<0.05$; Figure 6B), and a positive correlation with TSP1 ( $\mathrm{r}=0.63$; $\mathrm{p}$ value $<0.01)$ and PARVB $(\mathrm{r}=0.61$; $\mathrm{p}$ value $<0.01)$

\subsection{Plasma analyses of DIDO1 and FERM3}

In order to study a potential reflection of the expression of these proteins within thrombi with an increase of their circulating levels, DIDO1 and FERM3 proteins were analyzed by WB in immunodepleted plasma from STEMI patients versus healthy subjects. FERM3 showed a moderate non-significant increase in the plasma of STEMI patients $(\mathrm{p}$ value $=0.34$, Supplementary Figure 4B). DIDO1 WB of immunodepleted plasma showed 4 reactive bands for this protein, ranging from $60 \mathrm{kD}$ (molecular weight of DIDO1 isoform 2, which corresponds to the so-called DIDO1 species) to $40 \mathrm{kD}$ (Figure 6C). Previous optimization WB analyses performed platelet extracts also showed a variety of protein species from DIDO1 (Figure 4A), which could correspond to physiologically generated cleavage products.

After quantification, the $60 \mathrm{kDa}$ DIDO1 band was found up-regulated in the 
plasma of STEMI patients $($ T-test $\mathrm{p}$-value $=0.024)$. Moreover, total protein amount of

469 DIDO1, calculated as sum off all detected bands, was significantly increased in the plasma from STEMI patients $($ T-test p-value $=0.036$, Figure 6D).

471

\section{DISCUSSION}

Understanding the nature of the complex biological processes present in a tissue or organism requires an in-depth analysis at the molecular level, in the search for key proteins involved. In this sense, the characterization of the proteome of the human coronary thrombus may be a fundamental key to elucidate the mechanisms involved in its formation and it might help us to understand the plaque rupture process leading to acute myocardial infarction.

In the present study we aimed to describe the protein content of coronary thrombus, employing three proteomic approaches (2-DE MALDI-MS/MS, 1-DE LCMALDI-MS/MS and LC-ESI-MS/MS). On the other hand, we provide for the first time a protocol for analyzing by SRM, in a single method, a sub-group of proteins of interest, and cellular (CD41, platelets; CD3, T lymphocytes; CD14, monocytes; CD19, B lymphocytes; neutrophil elastase and eosinophil peroxidase) and ECM biomarkers (fibrin) in thrombotic material, with the possibility to contextualize expression changes with thrombus composition. Furthermore, the detection of 14 proteins by SRM analysis not only represents a validation of the thrombus characterization methodology performed, but also points out the implication of such molecules in human coronary thrombus formation.

A total number of 708 proteins were identified within the human coronary thrombus. The three different approaches performed have been proved to be complementary, since each method exclusively identified a subset of proteins, which 
493

494

495

496

497

498

499

500

501

502

503

504

505

506

507

508

509

510

511

512

513

514

515

516

517

were not found by the other two (Figure 4A). Although the 2-DE approach usually yields less number of identified proteins of a complex proteome than LC-based approaches, provides valuable information of observed molecular weight and isoelectric point, and potential posttranslational modifications. LC-MS/MS methodologies allowed identifying 138 common proteins, which could be considered as a "core proteome" of the human coronary thrombus from STEMI patients as a reference for future studies in the field.

Identified proteins were classified in seven different functional groups: "Cell differentiation", "Metabolism", "Redox state and apoptosis", "Regulation and transport", "Immune response and acute phase", "structural and cell adhesion", and "Other". The groups "Structural and cell adhesion" and "Regulation and transport" have a remarkable significance considering the percentage of proteins present in the thrombus. Both groups represent $62 \%$ of the total thrombus protein content, in which "cytoskeleton" and "intracellular signalling" proteins have a major contribution. Moreover, functional analysis performed using David 6.7 revealed a significant enrichment of "cytoskeleton" proteins in the thrombus, when Gene Ontology terms for molecular function and biological process were selected (Supplementary Figure 1). Pathway analysis using KEGG Database pointed out an outstanding implication of these proteins in focal adhesion (Figure 5) and various types of cell junctions related to cellcell and cell-matrix interactions (Supplementary Figure 2).

The group "Structural and cell adhesion" is represented both by cell membrane and cytoplasmic proteins. Among these identified proteins, a wide variety of integrins were found, like integrin alpha-IIb (CD41), which have a determinant role in platelet formation and activation [12]. Besides, correlation analyses showed a co-expression of a subset of 5 proteins (FERM3, MYH9, TSP1, PARVB and RAP1B) with the platelet 
marker CD41, which are indeed implicated in the aforementioned focal adhesion pathway (proteins are highlighted in red in Figure 5). TSP1 is a matricellular protein and a major component of $\alpha$-granules from platelets, which is released upon their activation [25]. After its release by platelets, contributes to cell adhesion through

522 binding the integrins that mediate platelet/platelet and platelet/matrix interactions (i.e.

523 CD41) [26]. Fermitin family homolog 3 (also called kindlin-3) is involved in adhesion 524 of hematopoietic cells, especially platelets [27], and regulates NF-kappa B expression and cell apoptosis [28]. Although its expression by platelets, hereby corroborated by SRM, has been found in a mouse model to be essential for aggregation and integrin activation [27], there were no previous evidences of the implication of this protein in platelet activation leading to thrombus formation. WB of immunodepleted plasma allowed detecting this protein and showed a moderate increase in STEMI patients, although statistical analysis did not find significant results. intracellular signalling mediated by ILK, therefore controlling cell adhesion, cell spreading, establishment or maintenance of cell polarity, and cell migration $[29,30]$.

534 This protein has been proved to be essential for platelet adhesion and spreading [31], but hereby we report for the first time its direct implication in such processes within the human thrombus. Myosin-9 plays an important role in cytoskeleton reorganization, focal adhesion and lamellipodial retraction during cell spreading [32]. Disruption of this

538 protein in mice showed a strong impair of thrombus growth and organization [33],

539 which is consistent with our results pointing out an outstanding role of MYH9 in 540 platelet activation during human coronary thrombus formation.

541 The first functional group in terms of number of proteins present is "Regulation 542 and transport", which is composed of several G-proteins and Ras-related proteins, 
543 protein families deeply involved in signal transduction. Functional analysis therefore

544 reported a significant enrichment of nucleotide binding proteins in the thrombus

545 proteome, corresponding to intracellular trafficking and ATP modulating molecules.

546 Among this group, ras-related protein Rap-1b participates to the conversion of integrins

547 into a high-affinity state for their ligands, that in turn favors platelet/platelet and platelet

548 /ECM interaction [34], and its implication in thrombosis has been proved in several in

549 vivo animal models [35,36]. Though, in this work we provide evidences of the

550 participation of RAP1B in platelet aggregation and adhesion to ECM within the

551 coronary thrombus.

552

This sub-group of adhesion proteins co-expressed with CD41 might be expressed

553

554

555

556

557

558

559

560

561

562

563

564

565

566

567

at early stages of thrombus formation, since platelet number decreases with ischemia time, as reported by Silvain et al. [11]. Further analyses are needed to establish a direct connection of these proteins with ischemia time.

Multimerin-1 acts as a carrier for coagulation factor $\mathrm{V}$ and it is stored by platelets in the $\alpha$-granules [37]. After released by these granules upon activation, MMRN1 is key to platelet aggregation and thrombus formation, as reported in mice studies [38]. Our results show a positive correlation of MMRN1 with both neutrophils and eosinophils and a negative correlation with monocytes (close to signification, $r=-0.42 ; \mathrm{p}$ value $<0.088$ ) and T-cells, but surpsisingly no correlation with platelet number was found. MMRN1 has been reported to be expressed by human leukocytes [39] but is mainly expressed by platelets and determinant in their aggregation and adhesion with neutrophils and endothelial cells [37, 40] (the protein is highlighted in blue in Figure 5). Although correlation of MMRN1 levels with neutrophil and eosinophil cell number could be explained by a specific expression of this protein by these cell types, reported expression of MMRN1 by leukocytes is minoritary and may not have driven such 
protein changes. Otherwise, release of MMRN1 by platelet $\alpha$-granules may be triggered

569

570

571

572

573

574

575

576

577

578

579

580

581

582

583

584

585

586

587

588

589

590

591

592

by neutrophils and/or eosinophils due to cytokine activation. Whether MMRN1 release

is activated by such cells or not, activated neutrophils adhere to this protein [40], and

thus expression of MMRN1 within the thrombus may provoke recruiting of neutrophils,

which may account for the observed positive correlation. Hence, eosinphils may also be recruited by MMRN1. On the other hand, monocyte and T-cell infiltrate may inhibit MMRN1 expression in the coronary thrombus, although there is no evidence of such mechanism in the literature and further analyses should be performed to prove this hypothesis.

The functional group "Metabolism" is mainly constituted by proteins implicated in energy metabolism. Pathway analysis also reported a significant enrichment of proteins from the metabolic route of Glycolisis/Gluconeogenesis. An important abundance of such proteins within the thrombus may come from an important contribution of this route to the energetic need triggered by the thrombotic process and the recruitment of circulating cells during its development [41]. Moreover, ATP binding proteins are significantly enriched in thrombus proteome, according to the functional analysis performed. The expression of a sub-group of enzymes implicated in redox activity, identified in the proteomic characterization of human coronary thrombus (carbonic anhydrases 1, 2 and 3; and flavin reductase (NAPDH)) was validated by SRM analysis in the studied thrombi. No correlation of this proteins with cell markers was found, which may imply they are present in several cell types with a similar expression pattern.

Concerning cell markers evaluated by SRM, infiltrated monocytes, T-cells and Bcells are simultaneously present within the thrombus, as stated by correlation analyses. Besides, neutrophils and eosinophils also co-localize in the human coronary thrombus. 
On the other hand, there is a negative correlation of neutrophils with monocytes and

CD3, which may highlight a distinct infiltration pattern of both co-localizing sub-groups

of leukocytes. A negative correlation of fibrin with $\mathrm{CD} 3$ was found, which may indicate a decrease of T-cells in older thrombi, since fibrin increases with ischemia time [11].

Other subset of proteins of interest, due to their implication in the thrombotic process, is the one related to "Redox state and apoptosis". Among these, catalase and

599 DIDO1 were analyzed by SRM to verify their expression in the thrombus and in the 600 search for correlations with cell markers and fibrin. DIDO1 is a pro-apoptotic transcription factor [42], which has not been previously reported to express in platelets, erythrocytes or blood plasma (according to HPRD database, www.hprd.org). WB analysis of platelet extract allowed verifying its actual expression by this cell type (Supplementary Figure 4A). Both WB and SRM analyses showed the expression of this protein during thrombogenesis. Moreover, a significant negative correlation of DIDO1 with fibrin was found (Figure 6B), which highlights a role of this protein in the early stages of thrombus development, since contribution of fibrin to thrombus composition increases with ischemia time [11]. Besides, DIDO1 was found up-regulated in the plasma of these STEMI patients. This result shows a direct relation between the human coronary thrombus expression of DIDO1 and increased levels of such protein in the plasma of these STEMI patients. This may therefore imply a release of the protein by

612 the thrombus to the bloodstream, which may be useful for diagnostic purposes.

\section{Study limitations}

It is important to note that these results were performed with aspirated material obtained during angioplasty and it is unclear to what extent this can be reliable/representative of the actual composition of a coronary thrombus, since aspiration of the thrombus may affect its protein content (51). On the other hand, drug 
anticoagulant therapies applied previous to angioplasty (pretereatment with ASA, clopidogrel in all patients) could also modify the thrombus proteome.

Preliminary results obtained for plasma levels of DIDO1, pointing to an upregulation of this protein with STEMI are promising but need to be further validated in a greater cohort of patients.

\section{CONCLUSSIONS}

The data hereby presented provide an in-depth characterization of the human coronary thrombus of STEMI patients and contributes to a better understanding of the mechanisms involved in the activation processes of platelets and other cell types implicated in thrombus formation leading to acute coronary syndrome. Moreover, the expression of DIDO1 within the human coronary thrombus has been associated with an increase of its plasma levels, which constitutes an important starting point for further quantification analyses of the proteins described in this work in the search for biomarkers of thrombosis.

\section{ACKNOWLEDGMENTS}

This work was supported by grants from the Instituto de Salud Carlos III (FIS PI070537, PI11/02239), Fondos Feder, Redes temáticas de Investigación Cooperativa en Salud (RD12/0042/0071, RD06/0014/1015), and Fundación para la Investigación Sanitaria de Castilla-La Mancha (FISCAM PI2008-08, PI2008-28, PI2008-52). These results are lined up with the Spanish initiative on the Human Proteome Project (SpHPP). The CNIC is supported by the Spanish Ministerio de Economia $y$ Competitividad and the Fundacion Pro-CNIC. We would like to thank Gemma Barroso from Proteomic Unit, Hospital Nacional de Paraplejicos, for her help and dedication to 
The authors have declared no conflicts of interests.

\section{FIGURE AND TABLE LEGENDS}

Figure 1. Flowchart showing the strategy applied for STEMI patients coronary thrombus characterization.

Figure 2. Representative histological analysis of coronary thrombus obtained by intracoronary thrombectomy. A, Low-augment view of a coronary thrombus (hematoxylin and eosin [H\&E] stain). B, Detail of panel A showing H\&E stain showing platelets, fibrin, erythrocytes and nucleated cells. C, Immunostaining with anti-CD41 antibody showing platelet presence in the thrombus. D, Immunostaining with anti-fibrinogen showing fibrin fibers and fibrinogen positive platelets. E, Neutrophil eleastase immunohistochemistry shows neutrophil infiltrate within the thrombus.

Figure 3. Representative 2-DE gel image from pooled human coronary thrombi. The analysis was performed with $17 \mathrm{~cm} \mathrm{IPG} \mathrm{strip,} \mathrm{pH} 4-7$ and SDS-PAGE $12 \%$ gels. Numbers correspond to the identified spots, as represented in Supplementary Table 3.

Figure 4. Characterization of the identified proteins. A, Venn diagram showing the number of common/exclusive proteins identified by every proteomic method. Functional classification (B) and Subcellular location $(\mathbf{C})$ of identified proteins.

Figure 5. Focal adhesion pathway and proteins with significant correlation. Focal adhesion pathway was found significantly represented on the thrombus proteome by pathway analysis performed with David Bioinformatics Resources 6.7 (NIH).

Symbols: 2 , proteins present and pathways significantly enriched in the thrombus proteome. $\longrightarrow$, molecular interaction or relation. $\rightarrow$, indirect effect. - , inhibition. $+\mathbf{p}$, phosphorylation, -p, dephosphorylation. A positive correlation of proteins highlighted with a red rectangle with CD41 was found. FERM3 is not a constituent of this KEGG Database pathway, but it is of the platelet activation one, and directly interacts with CD41 to induce platelet focal adhesion and therefore it was included in the represented scheme. Multimerin-1 (blue rectangle) was found to positively correlate with neutrohils and eosinophils. (Modified from KEGG Pathways Database, Kanehisa Laboratories)

Figure 6. Expression analyses and correlation with thrombus composition. Fourteen proteins were analyzed by SRM together with 6 cell markers and the ECM marker fibrin. A, Correlation analyses showed a positive correlation of 5 proteins associated with focal adhesion and platelet activation with CD41 (platelet marker): FERM3 ( $\mathrm{r}=0.94 ; \mathrm{p}$ value $<0.001)$, MYH9 ( $\mathrm{r}=0.89 ; \mathrm{p}$ value $<0.001)$, TSP1 ( $\mathrm{r}=0.96 ; \mathrm{p}$ value $<0.001)$ PARVB $(\mathrm{r}=0.96 ; \mathrm{p}$ value $<0.01)$ and RAP1B $(\mathrm{r}=0.95 ; \mathrm{p}$ value $<0.001))$. These proteins were all correlated with the other four too. $\mathbf{B}, \mathrm{A}$ negative correlation of DIDO1 protein with fibrin was also found $(r=-0.50, p$ value $<0.05)$. $\mathbf{C}$, Abundance of DIDO1 was investigated in plasma from the same STEMI patients from the thrombus analysis (STEMI: $n=17$, lanes 4-6, 10-12; control: $n=16$, lanes 1-3, 7-10). Both the complete protein of approximately $60 \mathrm{kDa}$ (dta not shown, pvalue $=0.024)$ and the sum of all bands $(\mathbf{D}$, pvalue $=0.036)$ observed (which may correspond to cleavage products of this protein) were over-expressed in STEMI patients' plasma, as observed after densitometry. OD*mm ${ }^{2}$, optical density per square millimetre.

Table 1. Clinical characteristics of STEMI patients recruited for human coronary thrombus proteomic analysis. 
688 Supplementary Figure 1. Summarized charts of David Bioinformatics Resources 6.7 689 software $(\mathrm{NIH})$ analyses performed with the 708 proteins identified for Gene Ontology 690 categories "molecular function" and "biological process".

691 Supplementary Figure 2. Most relevant functional pathways found significantly enriched in 692 human coronary thrombus proteome after bioinformatic analysis with David Bioinformatics 693 Resources 6.7 software.

694 Supplementary Figure 3. Extracted ion chromatograms of every peptide analyzed by SRM in 695 the optimization experiments, with its 3 monitored transitions.

696 Supplementary Figure 4. A, Western Blot analysis of DIDO1 showing specific expression by 697 platelets, with isoforms ranging from $65 \mathrm{kDa}$ to $40 \mathrm{kDa}$. B, Western Blot analysis of FERM3 in 698 immunodepleted plasma from STEMI patients versus healthy subjects. A moderate nonsignificant increase in STEMI patients can be observed ( $\mathrm{p}$ value $=0.34$ ).

Supplementary Table 1. Clinical characteristics of healthy controls employed for plasma Western Blot analysis.

Supplementary Table 2. Peptides and transitions used for SRM analysis of 14 proteins, 6 cell markers and fibrin.

Supplementary Table 3. Spots identified in 2-DE gel (pH: 4-7). Showing: accession number, theoretical and experimental isoelectric point and molecular weight, subcellular location (Cellular membrane: Cell mb; Cytoplasm: Cp; Nucleus: N; Nuclear membrane: $\mathrm{N}$ mb; Mitochondrial inner membrane: Mit inn mb; Secreted: Sec; Mitochondria: Mit; Extracellular space: ES; Melanosome: Mel; Peroxisome: Per; Lysosome: Lys; Golgi apparatus: Gol app; Endoplasmic Reticulum: ER; Podosome: Pod; Extracellular Matrix: EM) and primary function.

Supplementary Table 4. Proteins identified by 1-DE LC-MALDI-MS/MS. Showing: accession number, theoretical isoelectric point and molecular weight, subcellular location (Cellular membrane: Cell mb; Cytoplasm: $\mathrm{Cp}$; Nucleus: $\mathrm{N}$; Nuclear membrane: $\mathrm{N}$ mb; Mitochondrial inner membrane: Mit inn mb; Secreted: Sec; Mitochondria: Mit; Extracellular space: ES; Melanosome: Mel; Peroxisome: Per; Lysosome: Lys; Golgi apparatus: Gol app; Endoplasmic Reticulum: ER; Podosome: Pod; Extracellular Matrix: EM) and primary function.

Supplementary Table 5. Proteins identified by LC-ESI-MS/MS. Showing: accession number, theoretical molecular weight, subcellular location (Cellular membrane: Cell mb; Cytoplasm: $\mathrm{Cp}$; Nucleus: N; Nuclear membrane: N mb; Mitochondrial inner membrane: Mit inn mb; Secreted: Sec; Mitochondria: Mit; Extracellular space: ES; Melanosome: Mel; Peroxisome: Per; Lysosome: Lys; Golgi apparatus: Gol app; Endoplasmic Reticulum: ER; Podosome: Pod; Extracellular Matrix: EM) and primary function.

Supplementary Table 6. Unique proteins identified by the 3 proteomic approaches, proteins identified by both LC-MS/MS methods and subset identified by all 3 methods. Showing: accession number, molecular weight, subcellular location (Cellular membrane: Cell mb; Cytoplasm: Cp; Nucleus: $\mathrm{N}$; Nuclear membrane: $\mathrm{N} \mathrm{mb}$; Mitochondrial inner membrane: Mit inn mb; Secreted: Sec; Mitochondria: Mit; Extracellular space: ES; Melanosome: Mel; Peroxisome: Per; Lysosome: Lys; Golgi apparatus: Gol app; Endoplasmic Reticulum: ER; Podosome: Pod; Extracellular Matrix: EM) and primary function.

729 Supplementary Table 7. Pearson's correlation coefficient and $p$ value of all the significant and 
1 Libby P. Current concepts of the pathogenesis of the acute coronary syndromes. Circulation 2001;114: 365-372.

2 Libby P, Ganz P, Schoen FJ, Lee RT. The vascular biology of the acute coronary syndromes. In: Topol EJ, editor. Acute Coronary Syndromes. New York: Marcel Dekker, Inc.; 2000.pp.3357.

3 Barderas MG, Tuñón J, Dardé VM, De la Cuesta F, Durán MC, Jiménez-Nácher JJ, Tarín N, López-Bescós L, Egido J, Vivanco F. Circulating human monocytes in the acute coronary syndrome express a characteristic proteomic profile. J Proteome Res. 2007;6:876-886.

4 Huo Y, Schober A, Forlow SB, Smith DF, Hyman MC, Jung S, Littman DR, Weber C, Ley K. Circulating activated platelets exacerbate atherosclerosis in mice deficient in apolipoprotein $\mathrm{E}$. Nat Med. 2003;9:61-67.

5 Furman MI, Barnard MR, Krueger LA, Fox ML, Shilale EA, Lessard DM, Marchese P, Frelinger AL 3rd, Goldberg RJ, Michelson AD. Circulating monocyte-platelet aggregates are an early marker of acute myocardial infarction. J Am Coll Cardiol. 2001;38:1002-1006

6 Parguiña AF, Grigorian-Shamajian L, Agra RM, Teijeira-Fernández E, Rosa I, Alonso J, Viñuela-Roldán JE, Seoane A, González-Juanatey JR, García A. Proteins involved in platelet signaling are differentially regulated in acute coronary syndrome: a proteomic study. PLoS One. 2010;5:e13404

7 Svilaas T, van der Horst IC, Zijlstra F. Thrombus Aspiration during Percutaneous coronary intervention in Acute myocardial infarction Study (TAPAS) - Study design. Am Heart J. 2006;151:597.e1-597.e7

8 Li X, Kramer MC, VAN DER Loos CM, Ploegmakers HJ, DE Boer OJ, Koch KT, Tijssen JG, DE Winter RJ, VAN DER Wal AC. Early onset of endothelial cell proliferation in coronary thrombi of patients with an acute myocardial infarction: implications for plaque healing. $\mathrm{J}$ Thromb Haemost. 2012;10:466-73.

9 Kramer MC, van der Wal AC, Koch KT, Ploegmakers JP, van der Schaaf RJ, Henriques JP, Baan J Jr, Rittersma SZ, Vis MM, Piek JJ, Tijssen JG, de Winter RJ. Presence of older thrombus is an independent predictor of long-term mortality in patients with ST-elevation myocardial infarction treated with thrombus aspiration during primary percutaneous coronary intervention. Circulation. 2008;118:1810-6.

10 Yamashita A, Sumi T, Goto S, Hoshiba Y, Nishihira K, Kawamoto R, Hatakeyama K, Date $\mathrm{H}$, Imamura T, Ogawa H, Asada Y. Detection of von Willebrand factor and tissue factor in platelets-fibrin rich coronary thrombi in acute myocardial infarction. Am J Cardiol. 2006;97:2628.

11 Silvain J, Collet JP, Nagaswami C, Beygui F, Edmondson KE, Bellemain-Appaix A, Cayla G, Pena A, Brugier D, Barthelemy O, Montalescot G, Weisel JW. Composition of coronary thrombus in acute myocardial infarction. J Am Coll Cardiol. 2011;57:1359-67.

12 Huang H, Vogel HJ. Structural basis for the activation of platelet integrin $\alpha \operatorname{IIb} \beta 3$ by calciumand integrin-binding protein 1. J Am Chem Soc. 2012;134:3864-72.

13 Kaplan ZS, Jackson SP. The role of platelets in atherothrombosis. Hematology Am Soc Hematol Educ Program. 2011;2011:51-61.

14 García A, Prabhakar S, Brock CJ, Pearce AC, Dwek RA, Watson SP, Hebestreit HF, Zitzmann N. Extensive analysis of the human platelet proteome by two-dimensional gel electrophoresis and mass spectrometry. Proteomics. 2004;4:656-668.

15 Kakhniashvili DG, Bulla LA Jr, Goodman SR. The human erythrocyte proteome: analysis by ion trap mass spectrometry. Mol Cell Proteomics. 2004;3:501-509.

16 Jin M, Diaz PT, Bourgeois T, Eng C, Marsh CB, Wu HM. Two-dimensional gel proteome reference map of blood monocytes. Proteome Sci. 2006;4:16.

17 de la Cuesta F, Alvarez-Llamas G, Maroto AS, Donado A, Zubiri I, Posada M, Padial LR, Pinto AG, Barderas MG, Vivanco F A proteomic focus on the alterations occurring at the human atherosclerotic coronary intima. Mol Cell Proteomics. 2011;10:M110.003517. 
18 Lepedda AJ, Cigliano A, Cherchi GM, Spirito R, Maggioni M, Carta F, Turrini F, Edelstein C, Scanu AM, Formato M. A proteomic approach to differentiate histologically classified stable and unstable plaques from human carotid arteries. Atherosclerosis. 2009;203:112-118.

19 Guo W, Xue J, Shi J, Li N, Shao Y, Yu X, Shen F, Wu M, Liu S, Cheng S. Proteomics analysis of distinct portal vein tumor thrombi in hepatocellular carcinoma patients. J Proteome Res. 2010;9:4170-4175.

20 Moxon JV, Padula MP, Clancy P, Emeto TI, Herbert BR, Norman PE, Golledge J. Proteomic analysis of intra-arterial thrombus secretions reveals a negative association of clusterin and thrombospondin-1 with abdominal aortic aneurysm. Atherosclerosis. 2011;219:432-439.

21 de la Cuesta F, Barderas MG, Calvo E, Zubiri I, Maroto AS, Darde VM, Martin-Rojas T, Gil-Dones F, Posada-Ayala M, Tejerina T, Lopez JA, Vivanco F, Alvarez-Llamas G. Secretome analysis of atherosclerotic and non-atherosclerotic arteries reveals dynamic extracellular remodeling during pathogenesis. J Proteomics. 2012;75:2960-2971.

22 Martín-Rojas T, Gil-Dones F, Lopez-Almodovar LF, Padial LR, Vivanco F, Barderas MG. Proteomic profile of human aortic stenosis: insights into the degenerative process. J Proteome Res. 2012. 11:1537-1550.

23 Shevchenko A, Tomas H, Havlis J, Olsen JV, Mann M. In-gel digestion for mass spectrometric characterization of proteins and proteomes. Nat Protoc. 2006;1:2856-2860.

24 Huang DW, Sherman BT, Lempicki RA. Bioinformatics enrichment tools: paths toward the comprehensive functional analysis of large gene lists. Nucleic Acids Res. 2009;37:1-13.

25 Krishna SM, Golledge J. The role of thrombospondin-1 in cardiovascular health and pathology. Int J Cardiol. 2013;168:692-706.

26 Lawler J, Hynes RO. An integrin receptor on normal and thrombasthenic platelets that binds thrombospondin. Blood. 1989;1;74:2022-7.

27 Moser M, Nieswandt B, Ussar S, Pozgajova M, Fässler R. Kindlin-3 is essential for integrin activation and platelet aggregation. Nat Med. 2008;14:325-30.

28 Wang L, Deng W, Shi T, Ma D. URP2SF, a FERM and PH domain containing protein, regulates NF-kappaB and apoptosis. Biochem Biophys Res Commun. 2008;368:899-906.

29 Zhang Y, Chen K, Tu Y, Wu C. Distinct roles of two structurally closely related focal adhesion proteins, alpha-parvins and beta-parvins, in regulation of cell morphology and survival. J Biol Chem. 2004;279:695-705.

30 Kimura M, Murakami T, Kizaka-Kondoh S, Itoh M, Yamamoto K, Hojo Y, Takano M, Kario K, Shimada K, Kobayashi E. Functional molecular imaging of ILK-mediated Akt/PKB signaling cascades and the associated role of beta-parvin. J Cell Sci. 2010;123:747-55.

31 Randriamboavonjy V, Isaak J, Elgheznawy A, Pistrosch F, Frömel T, Yin X, Badenhoop K, Heide H, Mayr M, Fleming I. Calpain inhibition stabilizes the platelet proteome and reactivity in diabetes. Blood. 2012;120:415-23.

32 Betapudi V. Myosin II motor proteins with different functions determine the fate of lamellipodia extension during cell spreading. PLoS One. 2010;5:e8560.

33 Léon C, Eckly A, Hechler B, Aleil B, Freund M, Ravanat C, Jourdain M, Nonne C, Weber J, Tiedt R, Gratacap MP, Severin S, Cazenave JP, Lanza F, Skoda R, Gachet C. Megakaryocyterestricted MYH9 inactivation dramatically affects hemostasis while preserving platelet aggregation and secretion. Blood. 2007;110:3183-91.

34 Guidetti GF, Torti M. The Small GTPase Rap1b: A Bidirectional Regulator of Platelet Adhesion Receptors. J Signal Transduct. 2012;2012:412089.

35 M. Chrzanowska-Wodnicka, S. S. Smyth, S. M. Schoenwaelder, T. H. Fischer, and G. C. White, "Raplb is required for normal platelet function and hemostasis in mice," Journal of Clinical Investigation. 2005;115:680-687.

36 Stolla M, Stefanini L, Roden RC, Chavez M, Hirsch J, Greene T, Ouellette TD, Maloney SF, Diamond SL, Poncz M, Woulfe DS, Bergmeier W. The kinetics of $\alpha \mathrm{Ilb} \beta 3$ activation determines the size and stability of thrombi in mice: Implications for antiplatelet therapy. Blood. 2011;117:1005-1013.

37 Jeimy SB, Tasneem S, Cramer EM, Hayward CP. Multimerin 1. Platelets. 2008;19:83-95. 
38 Reheman A, Tasneem S, Ni H, Hayward CP. Mice with deleted multimerin 1 and alphasynuclein genes have impaired platelet adhesion and impaired thrombus formation that is corrected by multimerin 1 . Thromb Res. 2010;125:e177-83.

39 Raijmakers R, Heck AJ, Mohammed S. Assessing biological variation and protein processing in primary human leukocytes by automated multiplex stable isotope labeling coupled to 2 dimensional peptide separation. Mol Biosyst. 2009;5:992-1003.

40 Adam F, Zheng S, Joshi N, Kelton DS, Sandhu A, Suehiro Y, Jeimy SB, Santos AV, Massé JM, Kelton JG, Cramer EM, Hayward CP. Analyses of cellular multimerin 1 receptors: in vitro evidence of binding mediated by alphaIlbbeta3 and alphavbeta3. Thromb Haemost. 2005;945:1004-11.

41 Kim YW, West XZ, Byzova TV. Inflammation and oxidative stress in angiogenesis and vascular disease. J Mol Med (Berl). 2013;91:323-8.

42 Fütterer A, Campanero MR, Leonardo E, Criado LM, Flores JM, Hernández JM, San Miguel JF, Martínez-A C. Dido gene expression alterations are implicated in the induction of hematological myeloid neoplasms. J Clin Invest. 2005;115:2351-62. 
Demographics and risk factors

$$
\text { Age }
$$

Male

BMI

Dyslipidemia

Smoker

Diabetes

Hypertension

Past medical history of

ACS

$\mathrm{PCl}$

CABG

Clinical presentation

Anterior MI

TIMI risk score

Killip class

LVEF

Cardiogenic shock

Biomarkers on admission

Troponin I ( $\mu \mathrm{g} / \mathrm{ml})$

CK (IU/ml)

Troponin I maximum $(\mu \mathrm{g} / \mathrm{ml})$

CK maximum (IU/ml)

Fibrinogen $(\mathrm{g} / \mathrm{l})$

Platelets, $\mathrm{mm} 3$

Creatinine clearance $(\mathrm{ml} / \mathrm{min})$

Glycemia and lypidemia

Glucose $(\mathrm{mg} / \mathrm{ml})$

Cholesterol

Total $(\mathrm{mg} / \mathrm{ml})$

LDL $(\mathrm{mg} / \mathrm{ml})$

HDL $(\mathrm{mg} / \mathrm{ml})$

TG $(\mathrm{mg} / \mathrm{ml})$

Antithrombotic treatment

ASA

Clopidogrel

Heparin

UH

LMHW

GP Ilb/lia inhibitors
$59.8 \pm 11.1$

0.85

$27.7 \pm 3.5$

$40 \%$

$60 \%$

$10 \%$

$35 \%$

$10 \%$

$10 \%$

$5 \%$

$45 \%$

$24.2 \pm 10.5$

$1.1 \pm 0.3$

$46.4 \% \pm 10.0 \%$

$0 \%$

$16.9 \pm 26.6$

$997.1 \pm 1434.7$

$121.7 \pm 101.8$

$4167.4 \pm 7314.3$

$4.0 \pm 1.4$

$202.3 \pm 60.3$

$86.0 \pm 11.7$

$128.9 \pm 39.8$

$157.4 \pm 40.9$

$90.9 \pm 34.9$

$40.9 \pm 11.3$

$146.1 \pm 116.5$

$100 \%$

$100 \%$

$100 \%$

$0 \%$

$100 \%$ 
Click here to download high resolution image

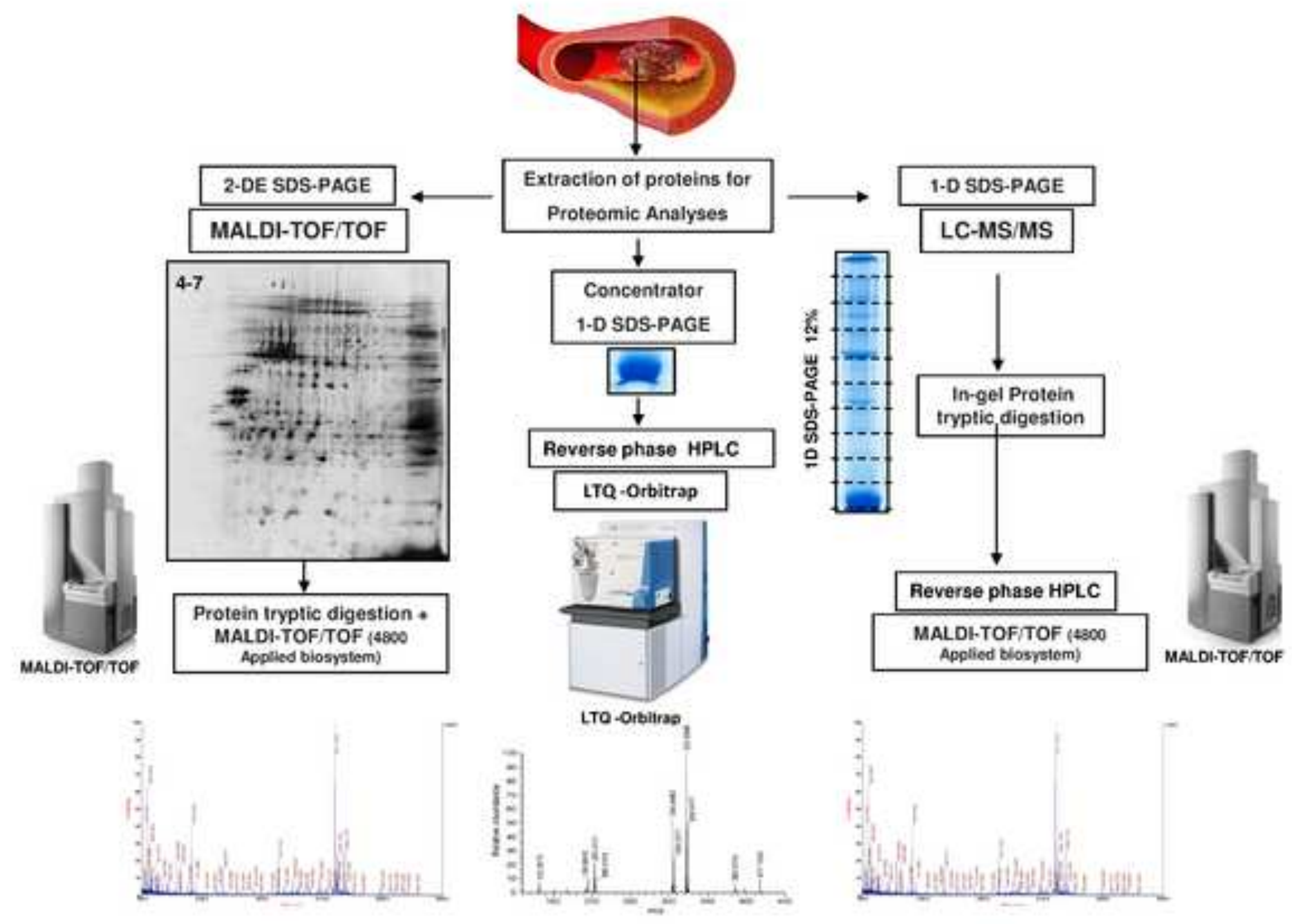




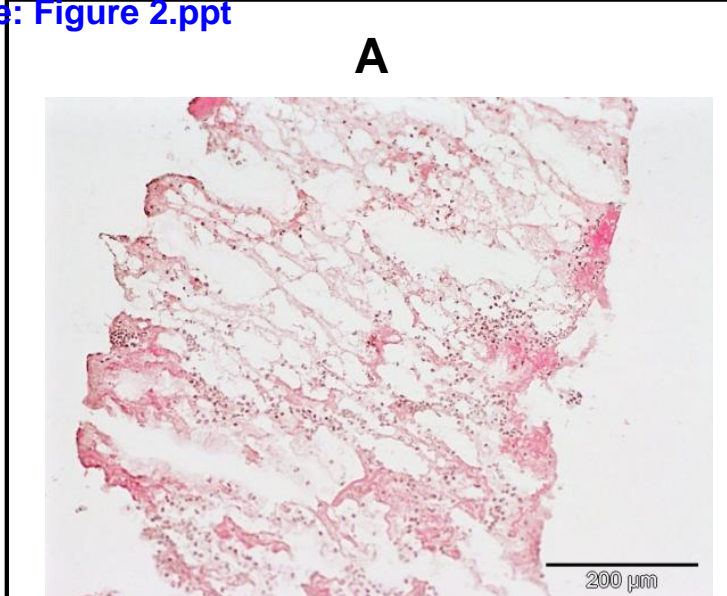

B
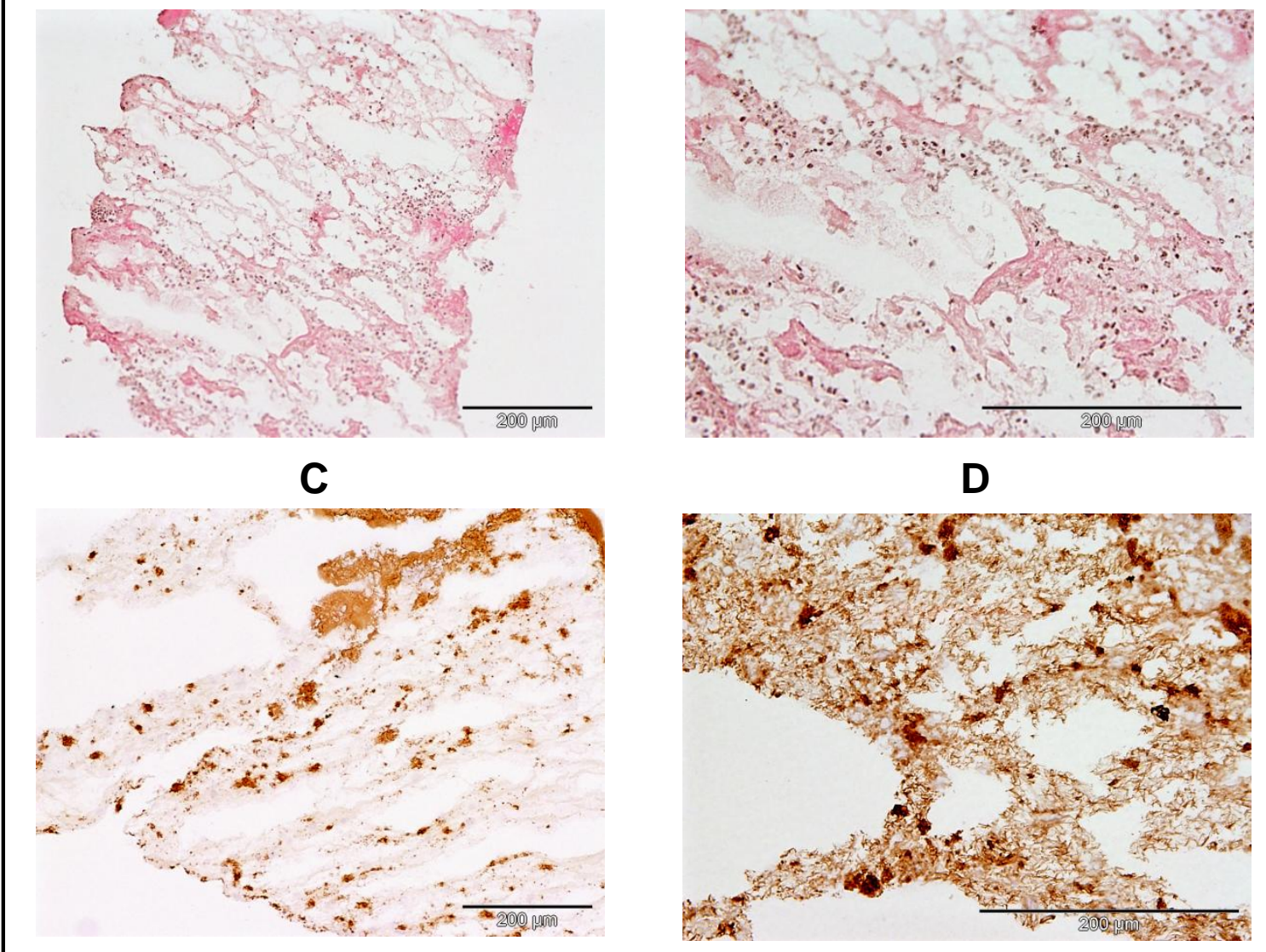

\section{D}
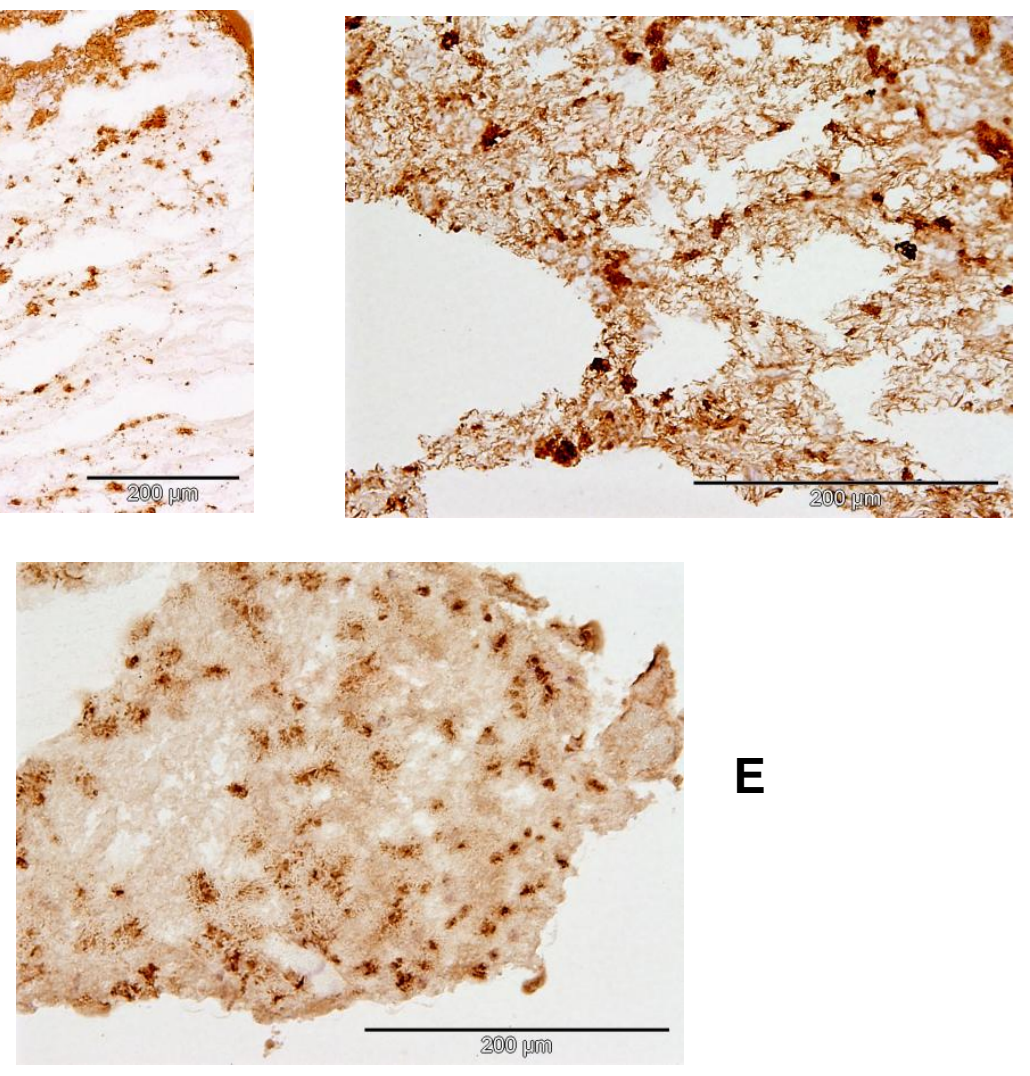

E 
Click here to download high resolution image

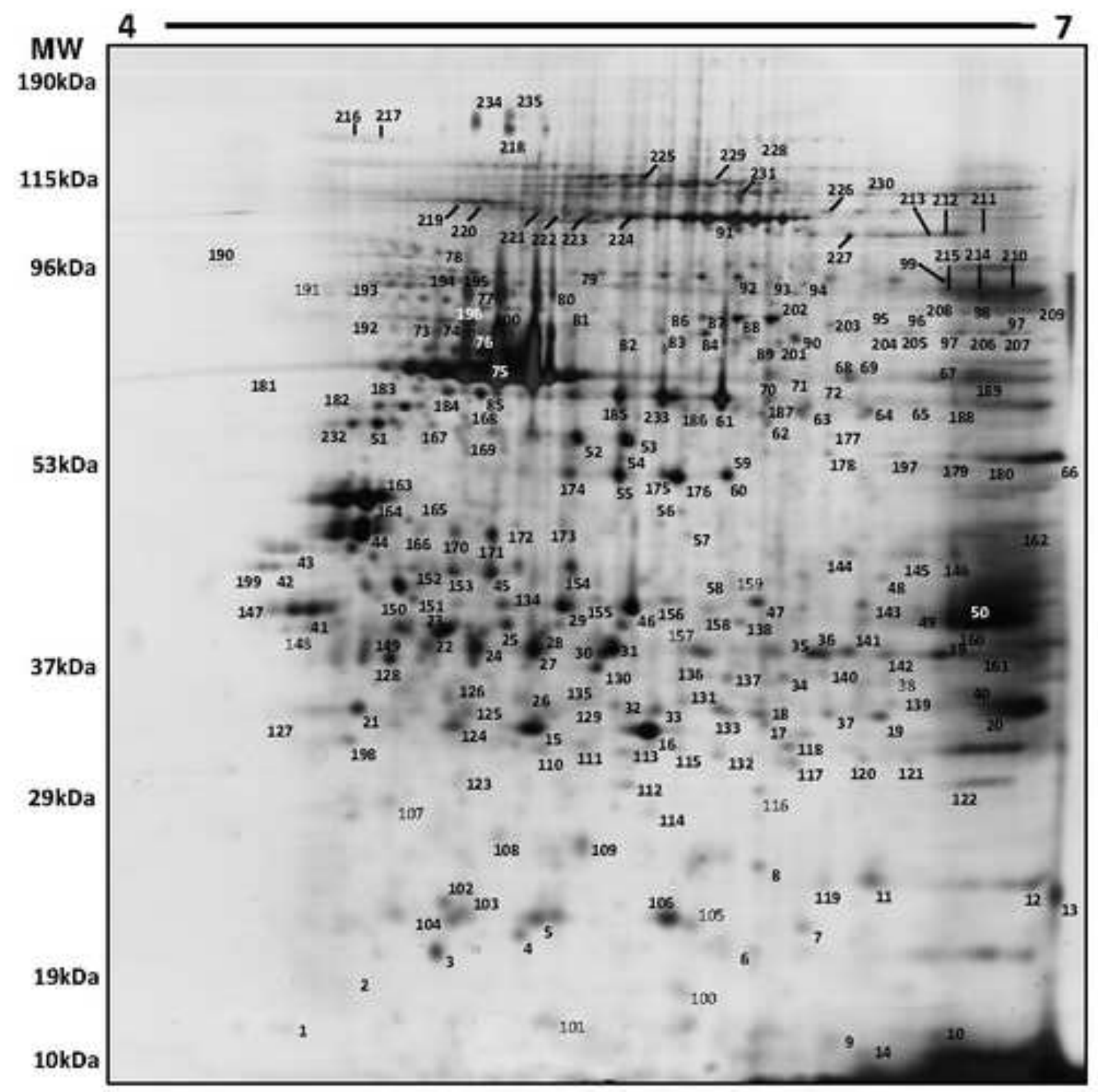


B

\section{Function}

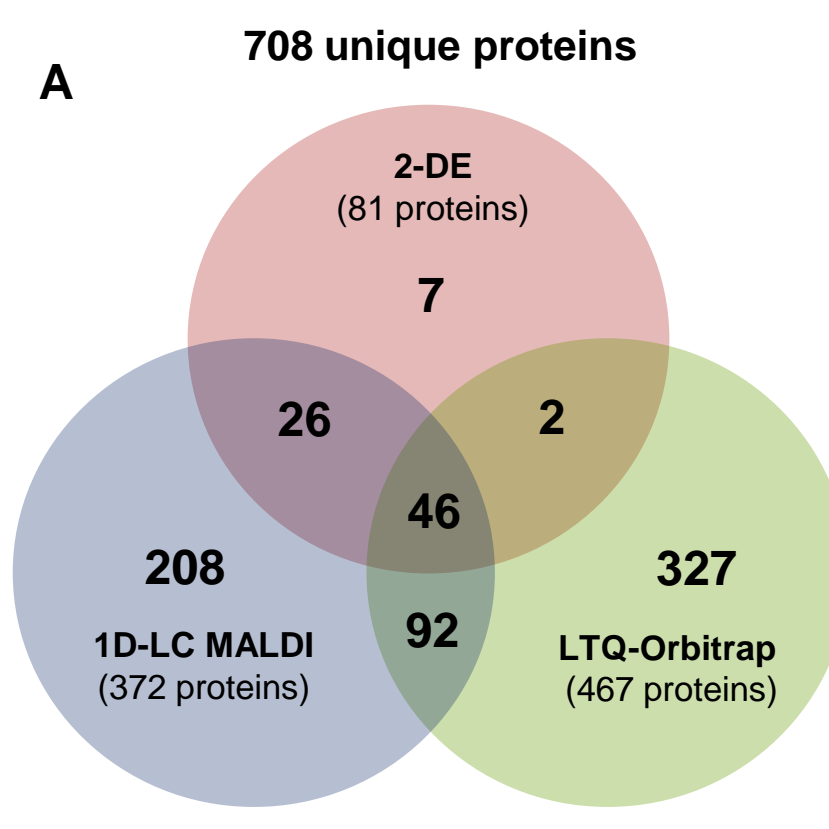

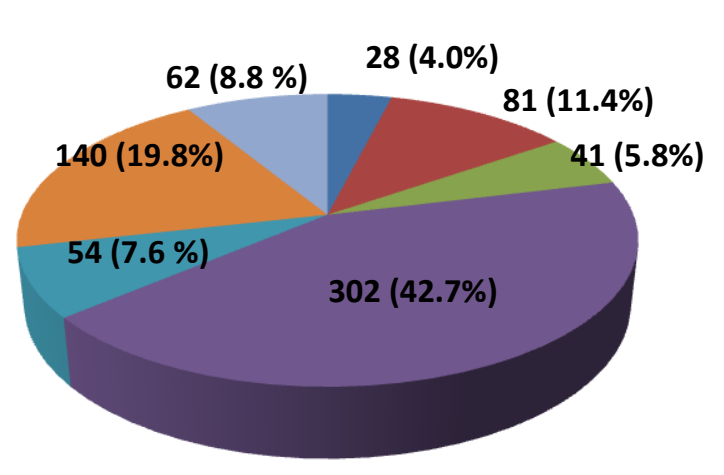

Cell differentiation

- Metabolism

- Redox state and apoptosis

- Regulation and transport

- Immune response-Acute phase

Structural and cell adhesion

- Other

\section{Subcellular Localization}

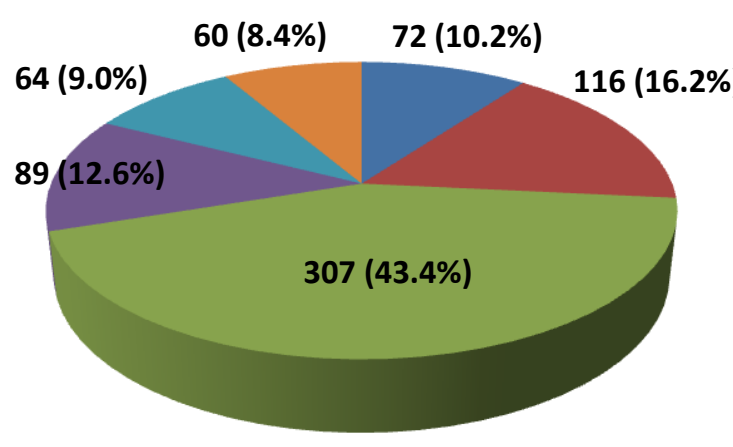

n Extracellular

- Cell membrane

Cytoplasm

nucleus

Others

Unknown 


\section{Figure 5}

Click here to download Figure: Figure 5.ppt

FOCAL ADHESION

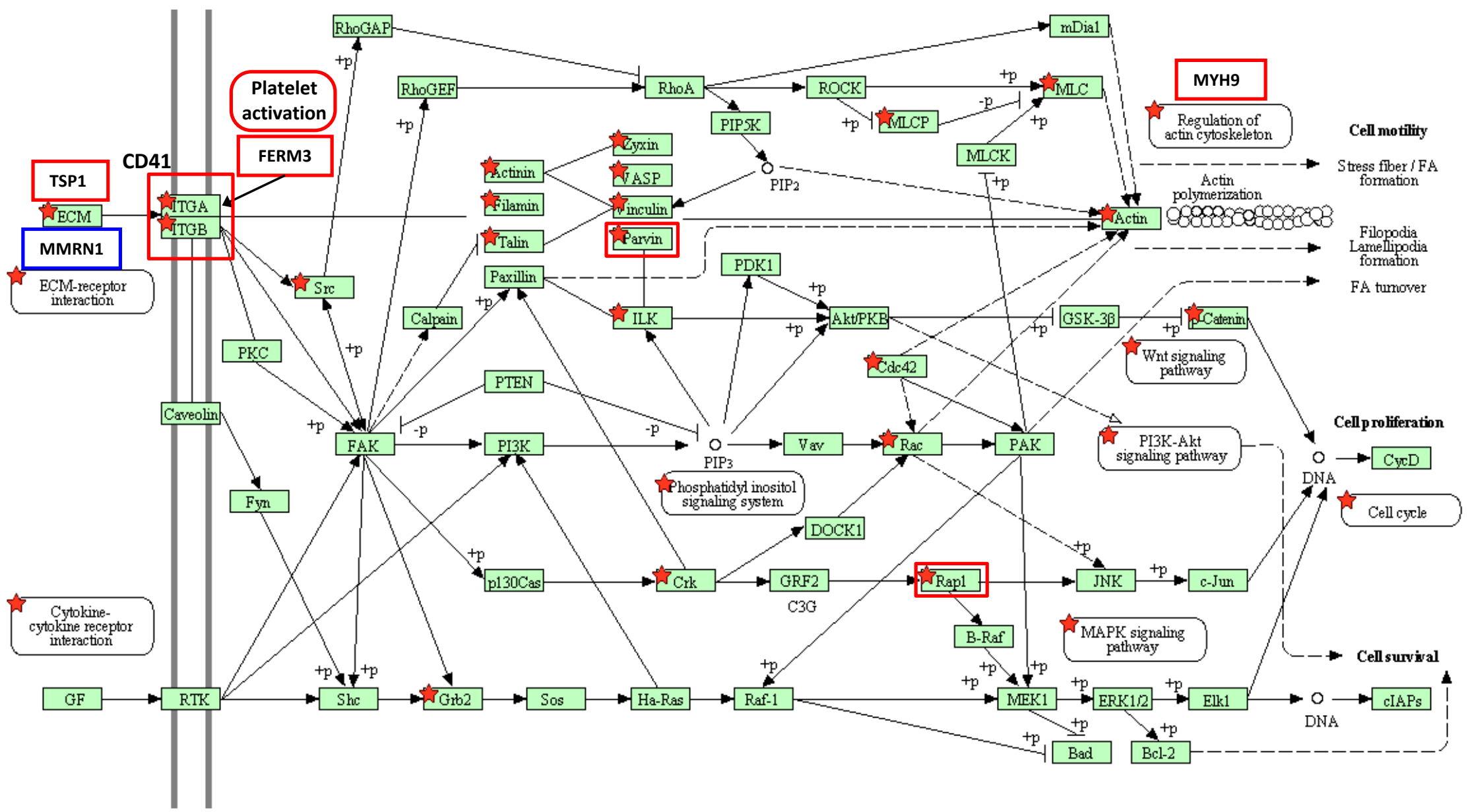

04510 10/2/12 
Click here to download Figure: Figure 6.ppt

A

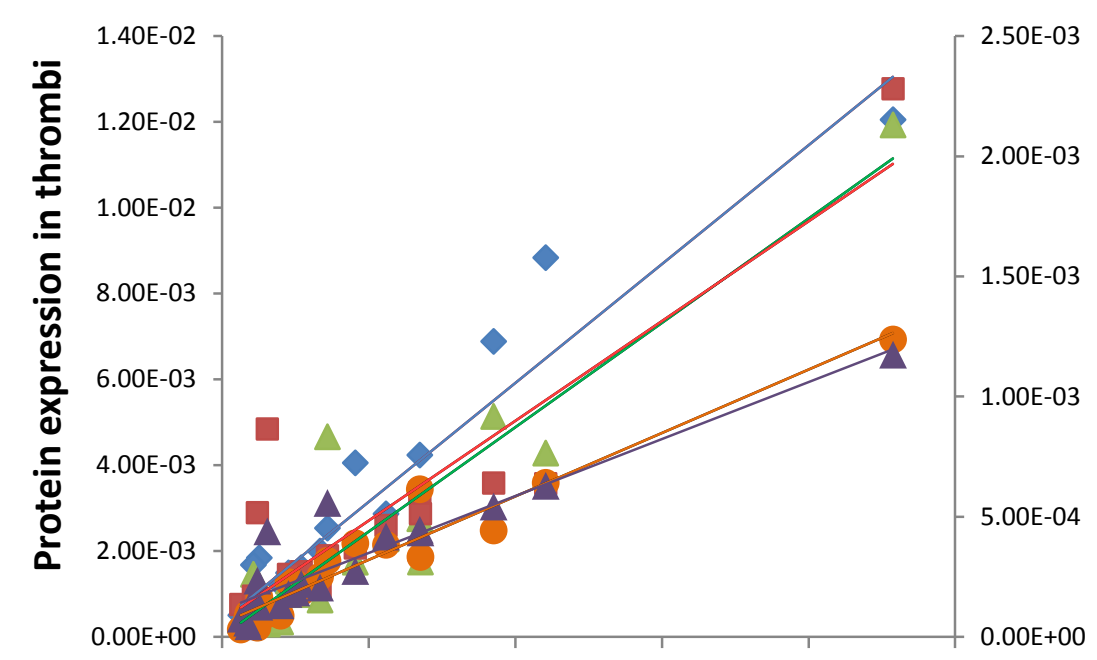

$0.00 \mathrm{E}+00 \quad 5.00 \mathrm{E}-03 \quad 1.00 \mathrm{E}-02 \quad 1.50 \mathrm{E}-02 \quad 2.00 \mathrm{E}-02 \quad 2.50 \mathrm{E}-02$

\section{CD41}

B

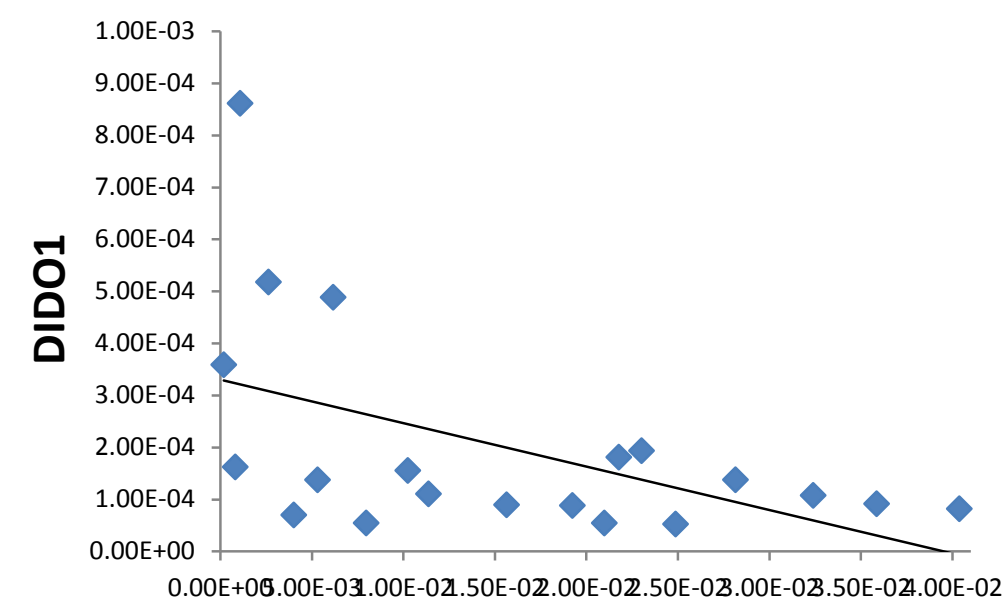

Fibrin

D

C

\section{$\triangle$ FERM3 \\ $\triangle \mathrm{TSP} 1$ \\ MYH9 \\ - PARVB \\ $\triangle$ RAP1B}

\section{$\begin{array}{llllllllllll}1 & 2 & 3 & 4 & 5 & 6 & 7 & 8 & 9 & 10 & 11 & 12\end{array}$}

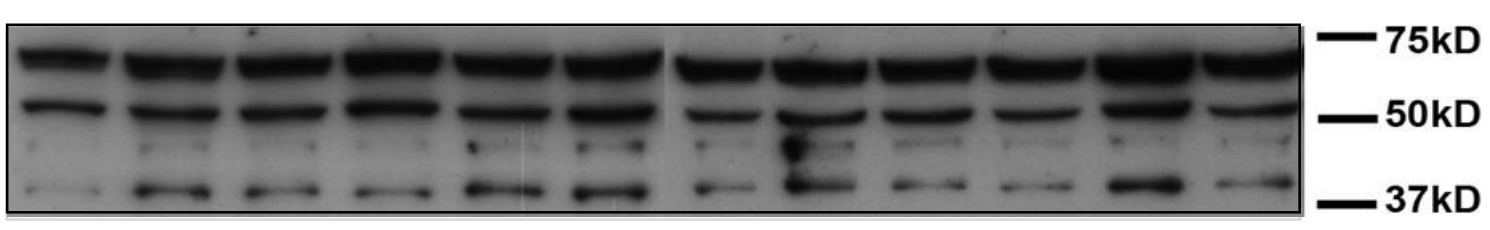

